\title{
Current surgical management of pelvic organ prolapse: Strategies for the improvement of surgical outcomes
}

\author{
Kwang Jin $\mathrm{Ko}^{1}$ (1), Kyu-Sung Lee ${ }^{2,3}$ (i) \\ 'Department of Urology, Hallym University Kangnam Sacred Heart Hospital, Hallym University College of Medicine, Seoul, ${ }^{2}$ Department of Urology, Samsung Medical \\ Center, Sungkyunkwan University School of Medicine, Seoul, 'Department of Medical Device Management and Research, SAIHST, Sungkyunkwan University, Seoul, \\ Korea
}

There are a variety of surgical management strategies to help surgeons repair pelvic organ prolapse (POP). Surgical treatment for POP includes native tissue repair, augmentation with mesh, and minimally invasive surgeries. Currently, laparoscopic or robotic techniques for POP repair are increasing in popularity and continuing to evolve. The aim of this review is to present an up-to-date review of surgical techniques used for POP repair and to discuss ways to optimize surgical outcomes.

Keywords: Cystocele; Pelvic organ prolapse; Review; Surgical mesh

This is an Open Access article distributed under the terms of the Creative Commons Attribution Non-Commercial License (http://creativecommons.org/licenses/by-nc/4.0) which permits unrestricted non-commercial use, distribution, and reproduction in any medium, provided the original work is properly cited.

\section{INTRODUCTION}

Pelvic organ prolapse (POP) is the descent of one or more of the anterior vaginal wall, posterior vaginal wall, the uterus, or the apex of the vagina (vaginal vault after hysterectomy) [1]. POP is seen on vaginal examinations in $40 \%$ to $60 \%$ of parous women; the most common sites for repair are the anterior and posterior compartments [2]. Women have an estimated $12.6 \%$ lifetime risk of undergoing POP repair [3]. Recent studies of POP surgery suggest that composite success should be evaluated not only through objective outcomes but also subjective symptomatic outcomes, reoperation rates, and complications. Due to the characteristics of POP, it is more important to improve patient satisfaction and reduce complications than to achieve anatomic success, considering concerns regarding native tissue repair and U. S. Food and Drug Administration (FDA) warnings. The popularity of minimally invasive surgery has increased the use of laparoscopic and robotic approaches in POP repair. The purpose of this article is to review the state of the art regarding surgery for POP.

\section{SURGERY TO TREAT ANTERIOR COMPARTMENT PROLAPSE}

There are various treatment of options for anterior compartment prolapse, including conservative management, pessaries, or surgical reconstruction. Unfortunately, there is no standard surgical treatment for anterior prolapse and it is crucial to discuss the risks and benefits of different surgical options with each patient. Generally, reconstruction of the anterior vaginal wall is performed by placing sutures

Received: 1 October, 2019 • Accepted: 22 October, 2019

Corresponding Author: Kyu-Sung Lee (iD https://orcid.org/0000-0003-0891-2488

Department of Urology, Samsung Medical Center, Sungkyunkwan University School of Medicine, 81 Irwon-ro, Gangnam-gu, Seoul 06351, Korea

TEL: +82-2-3410-3558, FAX: +82-2-3410-3027, E-mail: ksleedr@skku.edu 
that plicate and reduce the weakened tissues, and other native techniques have been introduced to further augment tissue and improve durability. Native tissue repair, although associated with lower success rates compared with meshaugmented repair, has been well-studied. Using current composite definitions of success, native repair is effective for relieving vaginal bulge symptoms and reducing prolapse within the vagina.

The success rate of POP surgery varies tremendously depending on the definition of treatment success that is used (19.2\%-97.2\%). Thus, it is difficult to compare outcomes because of variation among patients, surgical techniques, and definitions of success. The pelvic organ prolapse quantification (POPQ) system has been shown to be a valuable measurement tool that has improved our understanding of POP over the last 15 years and allowed reliable assessments of the anatomical success of POP surgeries. In 2001, an NIH workshop was held for the standardization of terminology between pelvic floor disorder researchers, where it was decided that the definition of "optimal anatomic outcome" requires perfect anatomic support (POPQ stage 0) and that the definition of "satisfactory anatomic outcome" requires support higher than $1 \mathrm{~cm}$ proximal to the hymen. "Cure" was defined as achieving an optimal or satisfactory anatomic outcome. More recently, it has been suggested that these anatomic definitions area are too strict, as more than $75 \%$ of women presenting for annual examinations without POP symptoms would not meet the criteria for "optimal anatomic outcome" and almost $40 \%$ of patients would not meet the criteria for "satisfactory anatomic outcome" [4]. Dividing stage 2 into stage $2 \mathrm{a}(-1 \mathrm{~cm}$ to hymen) and stage $2 \mathrm{~b}$ (hymen to $1 \mathrm{~cm}$ ) has also been considered. Recently, some studies have defined $\mathrm{Ba}$ point $<0$ as anatomical success, since the use of less stringent criteria to define "cure" are currently under discussion.

According to Barber et al. [5], the absence of vaginal bulge symptoms has the strongest relationship to patient assessments of overall improvement and treatment success. Additionally, definitions based on anatomic success had weak or no correlations with patient perceptions of outcomes. The goal of surgical repair is to maximize patient satisfaction, as well as return the pelvic organs to their original positions [6].

\section{Anatomic success}

Table 1 summarizes the results of a randomized controlled study comparing the results of anterior colporrhaphy (AC) to those of mesh repair over the last 10 years [7-23]. When anatomic success was defined as achieving a POPQ stage 0 or 1 , the results ranged from $39.5 \%$ to $75 \%$ for $\mathrm{AC}$ and $81.0 \%$ to $95 \%$ for mesh repair, with superior outcomes for mesh repair at 1-year follow-up [7,8,10,13,15-18]. In the mid-term follow-up (24-36 months), the results ranged from $39.5 \%$ to $86 \%$ for $\mathrm{AC}$ and $39.5 \%$ to $91.4 \%$ for mesh repair, indicating that mesh repair was superior in most studies $[9,12,14,20,22]$

Defining anatomic success as "No descent beyond the hymen $(\mathrm{Ba} \leq 0)$ " yields different results. In some studies, AC showed anatomic success rates as high as $86 \%$ to $89 \%$, which was not significantly different from the $84 \%$ to $96 \%$ success rates seen in mesh repair $[11,21,23]$. However, in patients with severe POP (POPQ stage 3-4), the success rate for mesh repair (86.4\%) was better than that for AC repair (70.4\%) $(p=0.019)[19]$.

\section{Symptomatic success}

Redefining the success of POP surgery has recently been suggested, and improvement of quality of life and patient satisfaction are now considered more important factors than anatomic success alone. The postoperative absence of vaginal bulge symptoms is significantly related to patient assessments of overall improvement and improvement in quality of life after surgery, while anatomic success alone does not ensure that vaginal bulge symptoms remain an important outcome assessment tool after POP surgery [5]. Several randomized trials have examined symptomatic success, using various measures such as the absence of vaginal bulge symptoms and several types of validated questionnaires (Table 1). When symptomatic success was defined as amelioration of vaginal bulge symptoms, symptoms remained in $0 \%$ to $37.9 \%$ of patients after $\mathrm{AC}$, and in $5 \%$ to $24.6 \%$ after mesh repair [8-11,14,17,18,21,22]. In half of the studies, the amelioration of bulging symptoms was superior to that in the mesh repair group, and the remaining studies did not reveal any significant differences between AC and mesh repair. A 2016 Cochrane review of anterior compartment prolapse reported that awareness of prolapse (risk ratio [RR], 0.56 ; $95 \%$ confidence interval [CI], 0.43-0.73) was significantly less common after mesh repair, compared to AC [24]. However, most questionnaires related to quality of life showed significant improvement after both treatments, but there was no significant difference in improvement between $\mathrm{AC}$ and mesh repair [7,12,13,19,20,23]. Although the anatomic success of AC is inferior to that of mesh repair, it has some advantages for quality of life. However, the use of mesh should be considered for treating anterior compartment prolapse in order to reduce the risk of recurrence and improve symptoms. 


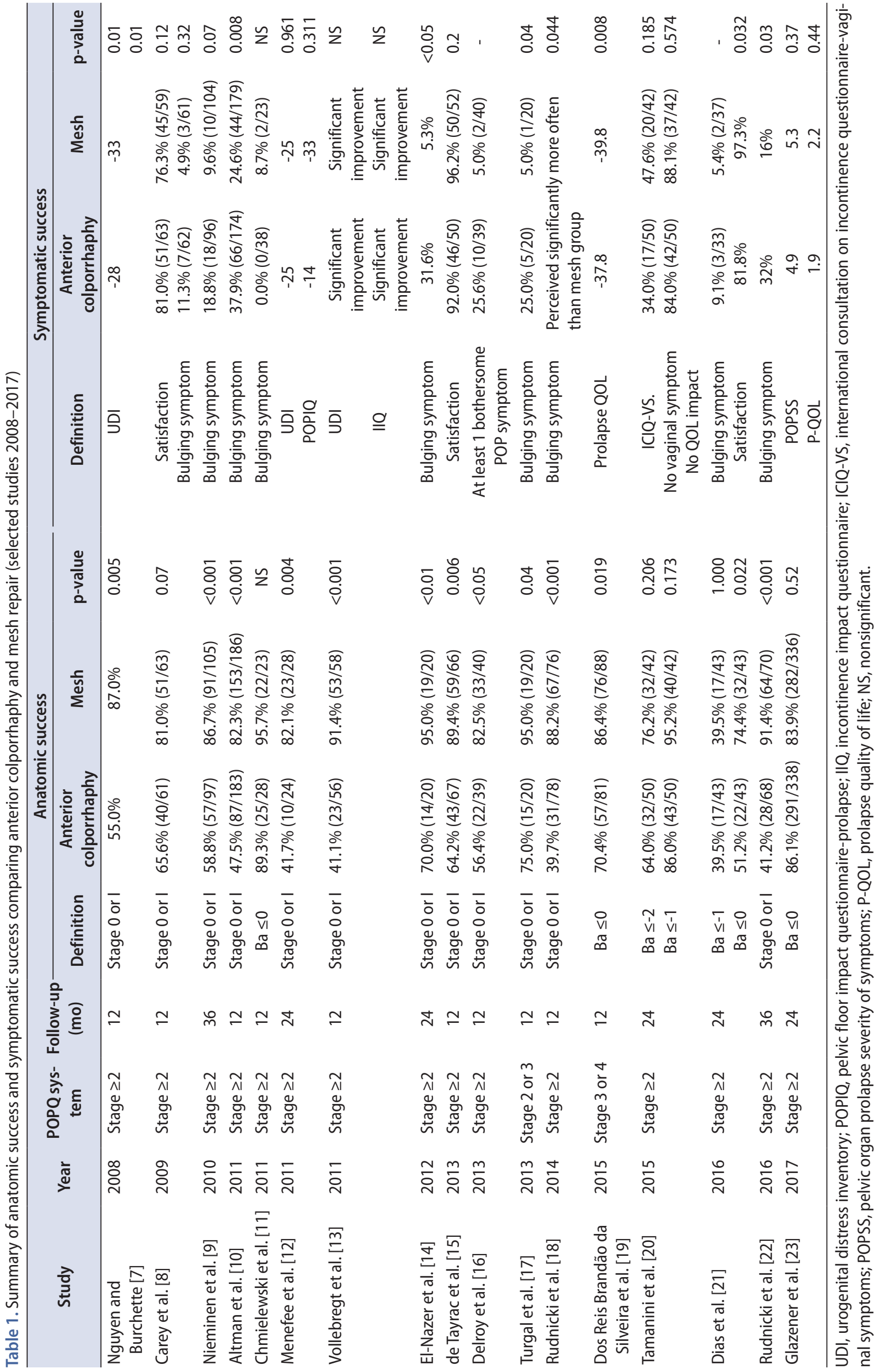




\section{Mesh issues: Is synthetic mesh really dangerous?}

The use of transvaginal mesh has been the subject of academic debate over the past decade. Vaginal mesh kits were first introduced in the USA in 2005 after being approved by the FDA in 2001 for POP repair. The vaginal mesh-kit is a simple device used for augmentation of native tissue in POP. Typically, the mesh consists of four arms and a main body that effectively covers both central defects and paravaginal defects. These standardized kits represent a departure from individualized assessments of patient anatomy [10]. Since their introduction into the market, the clinical use of meshkits has increased explosively, more quickly than evaluations of long-term safety have accumulated. In 2008, Sung et al. [25] reviewed studies comparing mesh use with native tissue for POP repair, and found that there was insufficient evidence to suggest that transvaginal mesh improves outcomes for POP. Adverse events associated with mesh were also evaluated, including erosion ( $0 \%-30 \%)$, visceral injury (1\%-4\%), urinary tract infection (0\%-19\%), and fistula formation (1\%). Overall, the results suggested that well-designed and adequately powered randomized trials are needed. Feiner et al. [26] also assessed success and complications in all trials to date that used transvaginal mesh and found that apical augmentation with transvaginal mesh resulted in good surgical outcomes with the most common complication being mesh erosion, occurring in $4.6 \%$ to $10.7 \%$ of patients. In 2008 , the FDA issued the first public health notification regarding complications associated with transvaginal mesh for POP repair. In 2009, Bako and Dhar [27] reported mesh erosion rates of $2 \%$ to $25 \%$ for anterior POP repair and mesh-related infection rates of up to $8 \%$. In 2011, the FDA issued a safety communication updating the 2008 notification, as follows [28].

- Recognize that in most cases POP can be treated successfully without mesh, thus avoiding mesh-related complications

- Choose mesh surgery only after weighing risks and benefits of surgery with mesh versus all surgical and nonsurgical alternatives

- Consider the following factors prior to placing mesh:

- Surgical mesh is a permanent implant that may make future repairs more challenging.

- A mesh procedure may put the patient at risk for requiring additional surgery or for the development of new complications.

- Removal of mesh may involve multiple surgeries and significantly impair the patient's quality of life.

- Complete removal may not be possible and may not result in resolution of complications, including pain.

- Mesh placed abdominally may result in lower rates of mesh complications compared with transvaginal mesh placement.

- Inform the patient about the benefits and risks of nonsurgical options, non-mesh surgery, abdominally placed mesh, and the likely success of these alternatives when compared with transvaginal mesh placement.

- Notify the patient if mesh will be used in her POP surgery and provide information about the specific product used.

- Ensure that the patient understands the postoperative risks and complications of mesh surgery and the limited long-term outcome data.

In response to increased reports of adverse events, the FDA concluded that transvaginal mesh has a higher complication rate than transabdominal mesh. In 2012, the FDA ordered mesh manufacturers to conduct post-market surveillance studies to evaluate efficacy and safety. In 2014, the FDA finally reclassified surgical mesh for POP as a class 3 (high-risk) device, with the reclassification formally implemented in January 2016. According to one study, vaginal mesh repairs decreased from 27\% of POP repairs before 2008 to $15 \%$ after the first FDA notification in 2008 and 5\% after the second notification in 2011, while the rates of minimally invasive surgeries such as laparoscopic or robotic sacral colpopexy or native tissue repair increased [29]. And finally, the FDA stopped selling transvaginal mesh for POP in April 2019. It does not apply to mesh products used to treat other conditions such as hernias or incontinence. The situation in Europe regarding the use of meshes in POP repair is no different. In 2014, the Medicines and Healthcare Products Regulatory Agency (MHRA) announced an official position on mesh for POP surgery, concluding that the use of mesh for POP surgery is safe and effective for the majority of patients, and that further research should be performed regarding implant types and surgical techniques. According to the official announcement of the National Institute for Health and Care Excellence (NICE) in April 2019, mesh should be considered as a final option for POP repair. In addition, when using a mesh, it is recommended that the patient be fully informed of outcomes and the possibility of mesh complication [30].

Is the use of synthetic mesh dangerous? It is important to not misunderstand the FDA's warning. Mesh exposure rates range from $3.2 \%$ to $20.5 \%$ according to recent randomized controlled studies and $3.1 \%$ to $14.4 \%$ according to singlearm studies. Mesh erosion rates vary between studies, so the risk of using synthetic mesh for POP cannot be determined. The mesh erosion rates for midurethral slings in 
patients with SUI also vary from study to study. In a study that analyzed 388 complications, the mesh erosion rate of the retropubic approach was $11.4 \%(24 / 210)$ and that of the transobturator approach was $25.7 \%$ (18/70) [31], which were moderately higher than the general mesh erosion rate of midurethral slings (3\%-5\%) [32]. Even when higher complication rates are reported, midurethral slings are not considered risky. In addition, when mesh exposure occurs, most cases are asymptomatic and conservative management can be expected to resolve symptoms. Patients who needed surgical treatment due to mesh exposure made up $0 \%$ to $16.4 \%$ of patients in randomized controlled trials [7-10,12-23] and 1.7\% to $8.9 \%$ in single-arm studies [33-37], accounting for only half of all patients with mesh exposure (Table 2). Comprehensively judging the recent literature and based on our experience, we suggest that how to select the proper patient is more important. Proper patient selection as well as improvements of surgical technique is essential for optimizing the success of mesh repair. Severe prolapse (stage 3 or 4), patient age younger than 60 years, diabetes mellitus, and recurrent prolapse are associated with recurrent prolapse after native tissue repair, and it is necessary to consider mesh repair

Table 2. Summary of mesh exposure rates and management after anterior compartment prolapse repair with mesh

\begin{tabular}{|c|c|c|c|c|c|}
\hline Study & Year & Follow-up (mo) & Exposure rate & Management & $\begin{array}{c}\text { Reoperation rate due to } \\
\text { mesh exposure }\end{array}$ \\
\hline \multicolumn{6}{|l|}{ Randomized control study } \\
\hline Nguyen and Burchette [7] & 2008 & 12 & $5.4 \%(2 / 37)$ & 2: conservative & $0.0 \%(0 / 27)$ \\
\hline Carey et al. [8] & 2009 & 12 & $5.8 \%(4 / 69)$ & $\begin{array}{l}\text { 3: surgical excision } \\
\text { 1: conservative }\end{array}$ & $4.3 \%(3 / 69)$ \\
\hline Vollebregt et al. [13] & 2011 & 12 & $3.4 \%(2 / 59)$ & 2: surgical excision & $3.4 \%(2 / 59)$ \\
\hline Menefee et al. [12] & 2011 & 24 & $13.9 \%(5 / 36)$ & $\begin{array}{l}\text { 3: conservative } \\
\text { 2: surgical excision }\end{array}$ & $5.6 \%(2 / 36)$ \\
\hline El-Nazer et al. [14] & 2012 & 24 & $5.0 \%(1 / 20)$ & 1: surgical excision & $5.0 \%(1 / 20)$ \\
\hline Delroy et al. [16] & 2013 & 12 & $5.0 \%(2 / 40)$ & 2: conservative & $0.0 \%(0 / 40)$ \\
\hline de Tayrac et al. [15] & 2013 & 12 & $9.3 \%(7 / 75)$ & $\begin{array}{l}\text { 1: conservative } \\
4: \text { surgical excision } \\
\text { 2: follow-up loss }\end{array}$ & $5.3 \%(4 / 75)$ \\
\hline Turgal et al. [17] & 2013 & 12 & $15.0 \%(3 / 20)$ & 3: surgical excision & $15.0 \%(3 / 20)$ \\
\hline Rudnicki et al. [18] & 2014 & 12 & $12.8 \%(10 / 78)$ & $\begin{array}{l}\text { 8: conservative } \\
\text { 2: surgical excision }\end{array}$ & $2.6 \%(2 / 78)$ \\
\hline Dos Reis Brandão da Silveira et al. [19] & 2015 & 12 & $20.5 \%(18 / 88)$ & $\begin{array}{l}\text { 15: conservative } \\
\text { 3: surgical excision }\end{array}$ & $3.4 \%(3 / 88)$ \\
\hline Altman et al. [10] & 2011 & 12 & $3.2 \%(6 / 186)$ & 6: surgical excision & $6.8 \%(6 / 88)$ \\
\hline Nieminen et al. [9] & 2010 & 12 & $20.8 \%(20 / 96)$ & $\begin{array}{l}\text { 6: conservative } \\
\text { 14: surgical excision }\end{array}$ & $14.6 \%(14 / 96)$ \\
\hline Tamanini et al. [20] & 2015 & 24 & $16.7 \%(7 / 42)$ & 7: surgical excision & $16.7 \%(7 / 42)$ \\
\hline Rudnicki et al. [22] & 2016 & 36 & $14.7 \%(10 / 68)$ & 10: conservative & $0.0 \%(0 / 68)$ \\
\hline Dias et al. [21] & 2016 & 24 & $11.6 \%(5 / 43)$ & $\begin{array}{l}\text { 3: conservative } \\
2 \text { : surgical excision }\end{array}$ & $4.7 \%(2 / 43)$ \\
\hline Glazener et al. [23] & 2017 & 24 & $5.7 \%(25 / 435)$ & $\begin{array}{l}\text { 17: surgical excision } \\
\text { 4: conservative } \\
\text { 4: no treatment }\end{array}$ & $3.9 \%(17 / 435)$ \\
\hline \multicolumn{6}{|l|}{ Single arm study } \\
\hline Jacquetin et al. [33] & 2010 & 40 & $14.4 \%(13 / 90)$ & $\begin{array}{l}\text { 8: surgical excision } \\
\text { 4: no treatment } \\
\text { 1: conservative }\end{array}$ & $8.9 \%(8 / 90)$ \\
\hline Bjelic-Radisic et al. [34] & 2014 & 19 & $12.1 \%(28 / 231)$ & $\begin{array}{l}\text { 21: conservative } \\
4: \text { surgical excision }\end{array}$ & $1.7 \%(4 / 231)$ \\
\hline Song et al. [35] & 2016 & 38 & $3.1 \%(5 / 163)$ & 5: surgical excision & $3.1 \%(5 / 163)$ \\
\hline Barski et al. [36] & 2017 & 12 & $5.9 \%(2 / 34)$ & $\begin{array}{l}\text { 1: surgical excision } \\
1: \text { conservative }\end{array}$ & $2.9 \%(1 / 34)$ \\
\hline Aubé et al. [37] & 2018 & 36 & $6.0 \%(20 / 334)$ & $\begin{array}{l}\text { 10: surgical excision } \\
\text { 10: conservative }\end{array}$ & $3.0 \%(10 / 334)$ \\
\hline
\end{tabular}


in these patients [38]. To date, known risk factors for mesh complications include diabetes, smoking, pelvic irradiation, and vaginal surgery [32]. In terms of surgical technique, full thickness vaginal wall dissection into the true vesicovaginal spaces is an integral technique for optimal mesh placement. The mesh should be placed flat without folding or excessive tension on the mesh.

\section{Importance of concurrent correction of apical prolapse}

The identification and correction of apical prolapse is critical to reduce recurrence after POP repair. Clinically significant apical prolapse is virtually always present in cases with both anterior and posterior compartment prolapse. If the anterior vaginal wall was at least $2 \mathrm{~cm}$ outside the hymen, $80 \%$ of the vaginal apices prolapsed to at least $2 \mathrm{~cm}$ inside the hymen, and $55 \%$ of the apices prolapsed $>2 \mathrm{~cm}$ outside the hymen [39]. Another study also found that almost $60 \%$ of patients with stage 2 or greater cystoceles had clinically significant apical vaginal descent. As cystocele stage increases, the predictive value of apical prolapse also increases [40]. A study of over 2,700 women comparing isolated anterior repair versus combined anterior and apical repair found that 10-year reoperation rates were lower in the combined anterior and apical repair group (11.6\% vs. 20.2\%) [41]. These findings are important as a basis for preventing recurrences, apart from a simple correlation between apical support and anterior support. Proper suspension of the vaginal apex is an essential factor for treatment success in cases of anterior compartment prolapse. Although concurrent apical repair is an obvious modifiable factor that can reduce the risk of recurrence, there are surgeons who perform anterior compartment repair without prior careful vaginal examination. According to US data, the proportion of anterior repairs without apical suspension decreased from $77.7 \%$ in 2004 to $41.4 \%$ in 2012 ( $p<0.001)$. Since 2011, there has been a decrease in the number of anterior repairs without apical suspension, notably among clinicians applying for Female Pelvic Medicine and Reconstructive Surgery (FPMRS) certification (17.1\% vs. $30.7 \%$ by all other urologists, $p<0.001$ ). Nonacademicallyaffiliated urologists are 2.1 times more likely to report anterior repair without apical suspension than academicallyaffiliated colleagues $(p<0.001)$ [42]. Therefore, it is necessary to improve POP repair practice through lifelong education, and surgeons who incapable of adequately assessing apical prolapse and correspondingly performing apical suspension should not attempt anterior compartment prolapse repair.

\section{SURGERY FOR APICAL VAGINAL PROLAPSE}

Apical prolapse surgeries can broadly be separated into obliterative and restorative approaches. Restorative approaches can be performed transvaginally or abdominally. For patients desiring restorative outcomes, abdominal sacrocolpopexy remains the gold standard. Abdominal sacrocolpopexy can be performed via laparotomy, conventional laparoscopic sacrocolpopexy (LSC), or robot assisted-laparoscopic sacrocolpopexy (RSC). In a recent Cochrane review [43], sacrocolpopexy including open or laparoscopic approaches were associated with lower risk of awareness of prolapse (RR, 2.11; 95\% CI, 1.06-4.21), recurrent prolapse (RR, 2.28; 95\% CI, 1.204.32 ), repeat surgery for prolapse (RR, 1.89; 95\% CI, 133-2.70), postoperative stress urinary incontinence (RR, 1.86; 95\% CI, 1.17-2.94) and dyspareunia (RR, 2.53; 95\% CI, 1.173-5.50) than a variety of vaginal approaches.

\section{Laparoscopic/robotic sacrocolpopexy vs. open sacrocolpopexy}

Although open sacrocolpopexy is a good treatment option for apical prolapse repair, with long-term success rates of $78 \%$ to $100 \%$, it is associated with increased length of hospital stay, analgesic requirements, and cost compared with transvaginal procedures [44,45]. New surgical techniques such as LSC or RSC have been developed to overcome these limitations. Compared with open sacrocolpopexy, LSC or RSC decreases overall morbidity and has good anatomical durability [46-52]. Freeman et al. [47] performed a randomized study comparing open sacrocolpopexy and LSC in patients with vault prolapse, and found that the methods demonstrated clinical similar recurrence rates at 1 year. In 2016, Costantini et al. [52] conducted the longest randomized follow-up study (mean follow-up of 41.7 months) comparing open sacrocolpopexy and LSC, and found that both techniques are efficacious with no patients in their sample experiencing apical recurrences. The 2016 Cochrane review [43] reported that there may be no difference between the results of LSC and open sacrocolpopexy for repeat surgery for prolapse (RR, 1.04; 95\% CI, 0.16-6.80).

Although LSC seems to overcome the shortcomings of open sacrocolpopexy, LSC is technically more challenging for those who are not proficient in laparoscopy. Since 2004, the implementation of RLC has allowed surgeons with good dexterity and precision to offer the procedure as an alternative to LSC. The learning curve is feasible without the need for laparoscopic skills. One of the largest prospective studies of RSC ( $n=120)$ showed that the anatomical success rate was 
89\% with 12-month follow-up [53]. According to a recent systematic review of LSC vs. RSC, RSC is associated with longer operation time, increased postoperative pain, and higher cost than LSC. However, both surgical options showed similar results regarding improvement of symptoms [54].

\section{Sacrohysteropexy for uterine preservation}

There are three options for the presence of prolapsed uterus in patients with apical prolapse: sacrohysteropexy, which fixes the uterus and vagina with a mesh to the sacral promontory, thereby preserving the uterus; supracervical hysterectomy with sacrocervicocolopopexy, which does not preserve the uterus; and sacrocolpopexy after total hysterectomy with closure of the vaginal cuff. Hysteropexy has the advantage of maintaining fertility and natural menopausal timing by preserving the uterus, and $36 \%$ to $60 \%$ of female patients choose uterine preservation assuming equal surgical efficacy. In addition, removal of the uterus may result in disruption of the uterosacral-cardinal ligaments and further weaken vaginal support. If there is no contraindication for uterine preservation, sacrohysteropexy may offer benefits. However, there is less surgical outcome data available for sacrohysteropexy, and the procedure requires continuous surveillance of the cervix and endometrium.

\section{1) Sacrohysteropexy vs. total hysterectomy and sacrocolpopexy}

There are no randomized trials comparing hysteropexy to hysterectomy and concurrent sacrocolpopexy. Costantini et al. [55] conducted prospective studies comparing abdominal sacrohysteropexy to total hysterectomy and sacrocolpopexy. In this study, 72 patients with grade 3 to 4 POP self-selected to undergo either sacrohysteropexy or total hysterectomy and sacrocolpopexy. Both groups demonstrated similar, good success rates $(100 \%$ and $100 \%)$ with no reoperations due to recurrence. The sacrohysteropexy group experienced shorter average operation time (89 vs. 115 minutes) and greater improvement in sexual function when compared to the total hysterectomy and sacrocolpopexy group. Another retrospective study comparing laparoscopic sacrohysteropexy $(n=65)$ to total laparoscopic hysterectomy and sacrocolpopexy demonstrated advantages in performing total hysterectomy and sacrocolpopexy [56]. The subjective satisfaction rate was significantly higher (92.3\% vs. $100 \%$, $\mathrm{p}<0.001)$ and the postoperative Pelvic Floor Impact Questionnaire $7(\mathrm{p}=0.043)$ and pelvic floor distress inventory-short form $20(\mathrm{p}=0.035)$ scores were significantly better in the total laparoscopic hysterectomy and sacrocolpopexy group, while the anatomical cure rates ( $72.3 \%$ vs. $88.2 \%$, $\mathrm{p}=0.07$ ) did not differ significantly between groups.

Based on current knowledge, there are significantly higher reoperation rates for POP in patients treated with hysteropexy, while the mesh exposure rate is 3.5 -fold higher after sacrocolpopexy among patients treated with concomitant total hysterectomy. It is difficult to determine whether sacrohysteropexy and total hysterectomy and sacrocolpopexy results in superior outcomes given our current knowledge [57-60].

\section{2) Supracervical hysterectomy and sacrocolpopexy}

The benefits of supracervical hysterectomy may reduce the risk of mesh erosion, thus avoiding cautery-induced thermal injury to the vagina [61]. Warner et al. [57] observed a $4.9 \%$ mesh exposure rate for the total hysterectomy group, but no mesh exposures were seen in the supracervical hysterectomy group $(p=0.03)$. However, evidence for the efficacy of supracervical hysterectomy is still lacking. A small study comparing laparoscopic sacrohysteropexy $(n=15)$ to laparoscopic sacrocolpopexy with concomitant supracervical hysterectomy showed that the overall success rate was significantly higher for laparoscopic supracervical hysterectomy with sacrocolpopexy ( $67 \%$ vs. $27 \%$ ), but major complications and vaginal mesh erosions were not registered [62]. A retrospective study demonstrated that supracervical hysterectomy with sacrocolpopexy was 2.8 times more likely to result in recurrent prolapse than total hysterectomy with sacrocolpopexy, when recurrent prolapse was defined as prolapse greater than or equal to stage 2 . This study did not have sufficient power to detect differences in rates of mesh exposure, with $7.5 \%$ in the total hysterectomy with sacrocolpopexy group vs. $2.3 \%$ in the supracervical hysterectomy with sacrocolpopexy group $(p=0.35)$ [61].

\section{Mesh fixation techniques}

In an effort to decrease the morbidity associated with open sacrocolpopexy, RSC, which decreases the difficulty associated with laparoscopic knot tying, and 3-dimensional visualization aiding sacral dissection, has rapidly gained popularity. However, variation in surgical technique includes the amount of vaginal dissection, type of mesh, number or location of sutures that should be placed to secure the suspending mesh, retroperitonealization of the mesh, and cervix preservation $[63,64]$.

\section{1) Absorbable vs. non-absorbable suture}

Traditional open sacrocolpopexy uses nonabsorbable suture to prevent the mesh detaching from the vagina and 
sacral promontory and to decrease the risk of mesh exposure and suture erosion. After synthetic mesh implantation, porcine models showed that $74 \%$ of the final strength of tissue ingrowth into mesh is achieved by 2 weeks, and maximum strength is reached by 3 months. Delayed absorbable monofilament suture lost $50 \%$ of its tensile strength by 4 weeks, $100 \%$ by 2 to 3 months, and was completely absorbed by 6 to 8 months [65]. In terms of risk of mesh complications, the mesh/suture exposure rate was 3.7\% (6/161) for braided non-absorbable suture (2-0 Ethibond; Ethicon, Somerville, NJ, USA) while no erosions occurred with monofilament delayed-absorbable suture (2-0 polydioxanone suture, Ethicon) $(p=0.002)$ [66]. In a series of RSC patients with median 33 months follow-up, the use of absorbable sutures for both vaginal and sacral mesh attachment was effective, with a 3-year rate of survival without repeat prolapse surgery of 93\%. However, in this study the benefit of risk of mesh erosion was not assessed [67]. Although evidence is lacking, it is unlikely that absorbable sutures are a risk factor for mesh detachment. Further studies will be needed to determine the proper location of sutures and the number of sutures, as well as the best type of suture to use in POP repair.

\section{2) Barbed suture}

The most challenging procedure during LSC or RSC is the attachment of the polypropylene mesh to the anterior and posterior vaginal walls and retroperitonealization over the mesh, which can be time-consuming. In LSC, suturing and knot tying are related to steeper learning curves compared to RSC [68-70]. To overcome this step, the use of barbed sutures has been described in several studies. Tan-Kim et al. [71] conducted a randomized study comparing non-barbed interrupted sutures to barbed suture (Quill ${ }^{\mathrm{TM}}$ ) for anchoring the mesh to the vaginal wall during LSC or RSC. Among all patients, those treated with non-barbed suture had significantly longer operation times than those treated with barbed suture (42 vs. 29 minutes, $p<0.001$ ) and there were no significant differences in anatomic failure between the groups at 12 months. Another retrospective study reported 1-year outcomes in 20 patients who underwent RSC using barbed delayed absorbable sutures (3-0 V-Loc 180, Covidien, Dublin, Ireland). The barbed delayed absorbable suture was used only for fixing the mesh to the vagina wall and performing retroperitonealization. There was no recurrence of apical prolapse or mesh exposure at 1-year follow-up [72]. Kallidonis et al. [73] also demonstrated that using barbed sutures for mesh fixation and peritoneal closure during LSC was safe and associated with reduced operating time. It is believed that the use of barbed suture plays reduces operation time by facilitating retroperitonealization of mesh. However, further studies should be performed for elucidating the ideal role of barbed suture for securing the mesh to the vaginal wall.

\section{Single port approach}

Single port approaches herald a new era in the field of minimally invasive surgery, with good cosmetic results and reduced patient morbidity compared with multiport surgery. Although single port robotic surgery is in an early stage of development, it has been implemented in various surgical fields. Since 2017, a few reports describing single port RSC have demonstrated that it is a feasible technique [74-77]. In 2017, Matanes et al. [76] reported their first 25 experiences with single port RSC and demonstrated significant decreases in median total operative and console times (226 minutes for the first 15 cases vs. 156 minutes for the next 10 cases), decreases that were within the same range as that reported for initial experiences with multiport access. There were no intraoperative adverse events. There was 1 case of small bowel obstruction that required reoperation, but this patient did not achieve retroperitonealized over the mesh, which altered the surgeon's approach toward all subsequent patients. Recently, Liu et al. [75] published a case series of patients treated with single port RSC following a modified technique. They attempted retroperitoneal tunneling techniques and asserted that they could more easily perform these techniques via a single port approach. A retroperitoneal tunnel was created by undermining the peritoneum with an articulated needle driver. The needle driver was placed in the peritoneal opening over the sacral promontory, and the tunnel was created just medial to the right uterosacral ligament in the direction of the vaginal vault by using forward pressure and a sweeping motion to create a space within the retroperitoneum. This approach allowed for easier adjustment and maintenance of mesh tension during the placement of sutures in the sacral promontory compared with opening the entire retroperitoneal space, and may reduce operative time and adhesion formation.

\section{SURGERY FOR POSTERIOR COMPARTMENT PROLAPSE}

Posterior vaginal wall prolapse can cause the sensation of bulging in the vagina and symptoms of obstructed defecation. The overall prevalence of posterior compartment prolapse alone is not certain, because it is usually accompanied by anterior or apical prolapse. As up to $80 \%$ of rectoceles are reported to be asymptomatic, its prevalence may be under- 
estimated [78]. Surgical treatment of posterior compartment prolapse can be approached either transvaginally, transperineally, or transanally, and can be repaired with native tissue or using mesh. Traditionally, central and lateral defects have been repaired with plication of rectovaginal fascia (known as posterior colporrhaphy). In 2006, Paraiso et al. [79] conducted a randomized study comparing outcomes of 3 different rectocele repair techniques: posterior colporrhaphy, site-specific repair, and site-specific repair augmented with a porcine small intestinal submucosal graft. After 1 year, subjects who received graft augmentation had a significantly greater anatomic failure rate $(12 / 26 ; 46 \%)$ than those who received site-specific repair alone $(6 / 27 ; 22 \%)$ or posterior colporrhaphy $(4 / 28 ; 14 \%)(\mathrm{p}=0.02)$. Although there are many studies of using mesh for posterior compartment repair, they do not separate the results of anterior and posterior repair. Anatomic success rates for posterior compartment repair with graft have ranged from $54 \%$ to $92 \%$, and have not been shown to have superior outcomes to native tissue repair. A 2018 Cochrane review identifying 10 randomized trials indicated that transvaginal repair may be more effective than other approaches for preventing recurrence of prolapse and that using mesh or graft materials offered no benefits for posterior compartment repair [80]. There does not appear to be controversy about posterior compartment prolapse repair anymore. We believe that the best option for treating posterior compartment prolapse is posterior colporrhaphy, which results in excellent outcomes with the lowest rates of recurrence.

\section{CONCLUSIONS}

In different cases, POP repair is achieved using slightly different techniques depending on the surgeon's skills. The definitions of treatment success and study designs are diverse in the literature, making it difficult to draw consistent conclusions. However, all previous research indicates that the goal of surgery is to maximize patient satisfaction as well as return the pelvic organs to their original positions. It is not an exaggeration to state that the success of POP repair is closely related to the experience of the surgeon, though the FDA warning against vaginal mesh has resulted in a decrease in the use of mesh. In addition, minimally invasive surgery has become more popular and is gradually evolving to be comparable to traditional techniques for POP repair.

\section{CONFLICTS OF INTEREST}

The authors have nothing to disclose.

\section{REFERENCES}

1. Haylen BT, de Ridder D, Freeman RM, Swift SE, Berghmans B, Lee J, et al.; International Urogynecological Association, International Continence Society. An International Urogynecological Association (IUGA)/International Continence Society (ICS) joint report on the terminology for female pelvic floor dysfunction. Neurourol Urodyn 2010;29:4-20.

2. Handa VL, Garrett E, Hendrix S, Gold E, Robbins J. Progression and remission of pelvic organ prolapse: a longitudinal study of menopausal women. Am J Obstet Gynecol 2004;190:27-32.

3. Wu JM, Matthews CA, Conover MM, Pate V, Jonsson Funk M. Lifetime risk of stress urinary incontinence or pelvic organ prolapse surgery. Obstet Gynecol 2014;123:1201-6.

4. Swift S, Woodman P, O'Boyle A, Kahn M, Valley M, Bland D, et al. Pelvic Organ Support Study (POSST): the distribution, clinical definition, and epidemiologic condition of pelvic organ support defects. Am J Obstet Gynecol 2005;192:795-806.

5. Barber MD, Brubaker L, Nygaard I, Wheeler TL 2nd, Schaffer J, Chen Z, et al.; Pelvic Floor Disorders Network. Defining success after surgery for pelvic organ prolapse. Obstet Gynecol 2009;114:600-9.

6. Lee U, Raz S. Emerging concepts for pelvic organ prolapse surgery: what is cure? Curr Urol Rep 2011;12:62-7.

7. Nguyen JN, Burchette RJ. Outcome after anterior vaginal prolapse repair: a randomized controlled trial. Obstet Gynecol 2008;111:891-8.

8. Carey M, Higgs P, Goh J, Lim J, Leong A, Krause H, et al. Vaginal repair with mesh versus colporrhaphy for prolapse: a randomised controlled trial. BJOG 2009;116:1380-6.

9. Nieminen K, Hiltunen R, Takala T, Heiskanen E, Merikari M, Niemi K, et al. Outcomes after anterior vaginal wall repair with mesh: a randomized, controlled trial with a 3 year follow-up. Am J Obstet Gynecol 2010;203:235.e1-8.

10. Altman D, Väyrynen T, Engh ME, Axelsen S, Falconer C; Nordic Transvaginal Mesh Group. Anterior colporrhaphy versus transvaginal mesh for pelvic-organ prolapse. N Engl J Med 2011;364:1826-36.

11. Chmielewski L, Walters MD, Weber AM, Barber MD. Reanalysis of a randomized trial of 3 techniques of anterior colporrhaphy using clinically relevant definitions of success. Am J Obstet Gynecol 2011;205:69.e1-8.

12. Menefee SA, Dyer KY, Lukacz ES, Simsiman AJ, Luber KM, Nguyen JN. Colporrhaphy compared with mesh or graftreinforced vaginal paravaginal repair for anterior vaginal wall prolapse: a randomized controlled trial. Obstet Gynecol 2011;118:1337-44.

13. Vollebregt A, Fischer K, Gietelink D, van der Vaart CH. Pri- 
mary surgical repair of anterior vaginal prolapse: a randomised trial comparing anatomical and functional outcome between anterior colporrhaphy and trocar-guided transobturator anterior mesh. BJOG 2011;118:1518-27.

14. El-Nazer MA, Gomaa IA, Ismail Madkour WA, Swidan KH, El-Etriby MA. Anterior colporrhaphy versus repair with mesh for anterior vaginal wall prolapse: a comparative clinical study. Arch Gynecol Obstet 2012;286:965-72.

15. de Tayrac R, Cornille A, Eglin G, Guilbaud O, Mansoor A, Alonso $S$, et al. Comparison between trans-obturator transvaginal mesh and traditional anterior colporrhaphy in the treatment of anterior vaginal wall prolapse: results of a French RCT. Int Urogynecol J 2013;24:1651-61.

16. Delroy CA, Castro Rde A, Dias MM, Feldner PC Jr, Bortolini MA, Girão MJ, et al. The use of transvaginal synthetic mesh for anterior vaginal wall prolapse repair: a randomized controlled trial. Int Urogynecol J 2013;24:1899-907.

17. Turgal M, Sivaslioglu A, Yildiz A, Dolen I. Anatomical and functional assessment of anterior colporrhaphy versus polypropylene mesh surgery in cystocele treatment. Eur J Obstet Gynecol Reprod Biol 2013;170:555-8.

18. Rudnicki M, Laurikainen E, Pogosean R, Kinne I, Jakobsson $\mathrm{U}$, Teleman P. Anterior colporrhaphy compared with collagencoated transvaginal mesh for anterior vaginal wall prolapse: a randomised controlled trial. BJOG 2014;121:102-10; discussion 110-1.

19. Dos Reis Brandão da Silveira S, Haddad JM, de Jármy-Di Bella ZI, Nastri F, Kawabata MG, da Silva Carramão S, et al. Multicenter, randomized trial comparing native vaginal tissue repair and synthetic mesh repair for genital prolapse surgical treatment. Int Urogynecol J 2015;26:335-42.

20. Tamanini JT, de Oliveira Souza Castro RC, Tamanini JM, Castro RA, Sartori MG, Girão MJ. A prospective, randomized, controlled trial of the treatment of anterior vaginal wall prolapse: medium term followup. J Urol 2015;193:1298-304.

21. Dias MM, de A Castro R, Bortolini MA, Delroy CA, Martins PC, Girão MJ, et al. Two-years results of native tissue versus vaginal mesh repair in the treatment of anterior prolapse according to different success criteria: a randomized controlled trial. Neurourol Urodyn 2016;35:509-14.

22. Rudnicki M, Laurikainen E, Pogosean R, Kinne I, Jakobsson U, Teleman P. A 3-year follow-up after anterior colporrhaphy compared with collagen-coated transvaginal mesh for anterior vaginal wall prolapse: a randomised controlled trial. BJOG 2016;123:136-42.

23. Glazener CM, Breeman S, Elders A, Hemming C, Cooper KG, Freeman RM, et al.; PROSPECT study group). Mesh, graft, or standard repair for women having primary transvaginal anterior or posterior compartment prolapse surgery: two parallel- group, multicentre, randomised, controlled trials (PROSPECT). Lancet 2017;389:381-92.

24. Maher C, Feiner B, Baessler K, Christmann-Schmid C, Haya N, Brown J. Surgery for women with anterior compartment prolapse. Cochrane Database Syst Rev 2016;11:CD004014.

25. Sung VW, Rogers RG, Schaffer JI, Balk EM, Uhlig K, Lau J, et al.; Society of Gynecologic Surgeons Systematic Review Group. Graft use in transvaginal pelvic organ prolapse repair: a systematic review. Obstet Gynecol 2008;112:1131-42.

26. Feiner B, Jelovsek JE, Maher C. Efficacy and safety of transvaginal mesh kits in the treatment of prolapse of the vaginal apex: a systematic review. BJOG 2009;116:15-24.

27. Bako A, Dhar R. Review of synthetic mesh-related complications in pelvic floor reconstructive surgery. Int Urogynecol J Pelvic Floor Dysfunct 2009;20:103-11.

28. Food and Drug Administration. Urogynecologic surgical mesh: update on the safety and effectiveness of transvaginal placement for pelvic organ prolapse. Food and Drug Administration; 2011.

29. Skoczylas LC, Turner LC, Wang L, Winger DG, Shepherd JP. Changes in prolapse surgery trends relative to FDA notifications regarding vaginal mesh. Int Urogynecol J 2014;25:471-7.

30. NICE Guidance - Urinary incontinence and pelvic organ prolapse in women: management: (C NICE (2019) Urinary incontinence and pelvic organ prolapse in women: management. BJU Int 2019;123:777-803.

31. Petri E, Ashok K. Comparison of late complications of retropubic and transobturator slings in stress urinary incontinence. Int Urogynecol J 2012;23:321-5.

32. MacDonald S, Terlecki R, Costantini E, Badlani G. Complications of transvaginal mesh for pelvic organ prolapse and stress urinary incontinence: tips for prevention, recognition, and management. Eur Urol Focus 2016;2:260-7.

33. Jacquetin B, Fatton B, Rosenthal C, Clavé H, Debodinance P, Hinoul $\mathrm{P}$, et al. Total transvaginal mesh (TVM) technique for treatment of pelvic organ prolapse: a 3-year prospective followup study. Int Urogynecol J 2010;21:1455-62.

34. Bjelic-Radisic V, Aigmueller T, Preyer O, Ralph G, Geiss I, Müller G, et al.; Austrian Urogynecology Working Group. Vaginal prolapse surgery with transvaginal mesh: results of the Austrian registry. Int Urogynecol J 2014;25:1047-52.

35. Song W, Kim TH, Chung JW, Cho WJ, Lee HN, Lee YS, et al. Anatomical and functional outcomes of prolift transvaginal mesh for treatment of pelvic organ prolapse. Low Urin Tract Symptoms 2016;8:159-64.

36. Barski D, Arndt C, Gerullis H, Yang J, Boros M, Otto T, et al. Transvaginal PVDF-mesh for cystocele repair: a cohort study. Int J Surg 2017;39:249-54.

37. Aubé M, Guérin M, Rheaume C, Tu LM. Efficacy and pa- 
tient satisfaction of pelvic organ prolapse reduction using transvaginal mesh: a Canadian perspective. Can Urol Assoc J 2018;12:E432-7.

38. Whiteside JL, Weber AM, Meyn LA, Walters MD. Risk factors for prolapse recurrence after vaginal repair. Am J Obstet Gynecol 2004;191:1533-8.

39. Rooney K, Kenton K, Mueller ER, FitzGerald MP, Brubaker L. Advanced anterior vaginal wall prolapse is highly correlated with apical prolapse. Am J Obstet Gynecol 2006;195:1837-40.

40. Elliott CS, Yeh J, Comiter CV, Chen B, Sokol ER. The predictive value of a cystocele for concomitant vaginal apical prolapse. J Urol 2013;189:200-3.

41. Eilber KS, Alperin M, Khan A, Wu N, Pashos CL, Clemens JQ, et al. Outcomes of vaginal prolapse surgery among female Medicare beneficiaries: the role of apical support. Obstet Gynecol 2013;122:981-7.

42. Liu JS, Nettey O, Vo AX, Hofer MD, Flury SC, Kielb SJ. Prolapse repair with and without apical resuspension-Practice patterns among certifying American urologists. Neurourol Urodyn 2017;36:344-8.

43. Maher C, Feiner B, Baessler K, Christmann-Schmid C, Haya N, Brown J. Surgery for women with apical vaginal prolapse. Cochrane Database Syst Rev 2016;10:CD012376.

44. Lee RK, Mottrie A, Payne CK, Waltregny D. A review of the current status of laparoscopic and robot-assisted sacrocolpopexy for pelvic organ prolapse. Eur Urol 2014;65:1128-37.

45. Nygaard IE, McCreery R, Brubaker L, Connolly A, Cundiff G, Weber AM, et al.; Pelvic Floor Disorders Network. Abdominal sacrocolpopexy: a comprehensive review. Obstet Gynecol 2004;104:805-23.

46. Anger JT, Mueller ER, Tarnay C, Smith B, Stroupe K, Rosenman A, et al. Robotic compared with laparoscopic sacrocolpopexy: a randomized controlled trial. Obstet Gynecol 2014; 123:5-12.

47. Freeman RM, Pantazis K, Thomson A, Frappell J, Bombieri L, Moran P, et al. A randomised controlled trial of abdominal versus laparoscopic sacrocolpopexy for the treatment of posthysterectomy vaginal vault prolapse: LAS study. Int Urogynecol J 2013;24:377-84.

48. Geller EJ, Siddiqui NY, Wu JM, Visco AG. Short-term outcomes of robotic sacrocolpopexy compared with abdominal sacrocolpopexy. Obstet Gynecol 2008;112:1201-6.

49. Nosti PA, Umoh Andy U, Kane S, White DE, Harvie HS, Lowenstein L, et al. Outcomes of abdominal and minimally invasive sacrocolpopexy: a retrospective cohort study. Female Pelvic Med Reconstr Surg 2014;20:33-7.

50. Paraiso MF, Jelovsek JE, Frick A, Chen CC, Barber MD. Laparoscopic compared with robotic sacrocolpopexy for vaginal prolapse: a randomized controlled trial. Obstet Gynecol
2011;118:1005-13.

51. Siddiqui NY, Geller EJ, Visco AG. Symptomatic and anatomic 1 -year outcomes after robotic and abdominal sacrocolpopexy. Am J Obstet Gynecol 2012;206:435.e1-5.

52. Costantini E, Mearini L, Lazzeri M, Bini V, Nunzi E, di Biase M, et al. Laparoscopic versus abdominal sacrocolpopexy: a randomized, controlled trial. J Urol 2016;196:159-65.

53. Salamon CG, Lewis C, Priestley J, Gurshumov E, Culligan PJ. Prospective study of an ultra-lightweight polypropylene Y mesh for robotic sacrocolpopexy. Int Urogynecol J 2013;24:1371-5.

54. Pan $\mathrm{K}$, Zhang Y, Wang Y, Wang Y, Xu H. A systematic review and meta-analysis of conventional laparoscopic sacrocolpopexy versus robot-assisted laparoscopic sacrocolpopexy. Int J Gynaecol Obstet 2016;132:284-91.

55. Costantini E, Porena M, Lazzeri M, Mearini L, Bini V, Zucchi A. Changes in female sexual function after pelvic organ prolapse repair: role of hysterectomy. Int Urogynecol J 2013;24:1481-7.

56. Pan K, Cao L, Ryan NA, Wang Y, Xu H. Laparoscopic sacral hysteropexy versus laparoscopic sacrocolpopexy with hysterectomy for pelvic organ prolapse. Int Urogynecol J 2016;27:93101.

57. Warner WB, Vora S, Hurtado EA, Welgoss JA, Horbach NS, von Pechmann WS. Effect of operative technique on mesh exposure in laparoscopic sacrocolpopexy. Female Pelvic Med Reconstr Surg 2012;18:113-7.

58. Tan-Kim J, Menefee SA, Luber KM, Nager CW, Lukacz ES. Prevalence and risk factors for mesh erosion after laparoscopic-assisted sacrocolpopexy. Int Urogynecol J 2011;22:205-12.

59. Stepanian AA, Miklos JR, Moore RD, Mattox TF. Risk of mesh extrusion and other mesh-related complications after laparoscopic sacral colpopexy with or without concurrent laparoscopic-assisted vaginal hysterectomy: experience of 402 patients. J Minim Invasive Gynecol 2008;15:188-96.

60. Meyer I, McGwin G, Swain TA, Alvarez MD, Ellington DR, Richter HE. Synthetic graft augmentation in vaginal prolapse surgery: long-term objective and subjective outcomes. J Minim Invasive Gynecol 2016;23:614-21.

61. Myers EM, Siff L, Osmundsen B, Geller E, Matthews CA. Differences in recurrent prolapse at 1 year after total vs supracervical hysterectomy and robotic sacrocolpopexy. Int Urogynecol J 2015;26:585-9.

62. Gracia M, Perelló M, Bataller E, Espuña M, Parellada M, Genís $\mathrm{D}$, et al. Comparison between laparoscopic sacral hysteropexy and subtotal hysterectomy plus cervicopexy in pelvic organ prolapse: a pilot study. Neurourol Urodyn 2015;34:654-8.

63. Lee W, Tam J, Kobashi K. Surgery for apical vaginal prolapse after hysterectomy: abdominal sacrocolpopexy. Urol Clin North Am 2019;46:113-21. 
64. Oliver JL, Kim JH. Robotic sacrocolpopexy-is it the treatment of choice for advanced apical pelvic organ prolapse? Curr Urol Rep 2017;18:66.

65. Majercik S, Tsikitis V, Iannitti DA. Strength of tissue attachment to mesh after ventral hernia repair with synthetic composite mesh in a porcine model. Surg Endosc 2006;20:1671-4.

66. Greenberg JA, Clark RM. Advances in suture material for obstetric and gynecologic surgery. Rev Obstet Gynecol 2009;2:146-58.

67. Linder BJ, Anand M, Klingele CJ, Trabuco EC, Gebhart JB, Occhino JA. Outcomes of robotic sacrocolpopexy using only absorbable suture for mesh fixation. Female Pelvic Med Reconstr Surg 2017;23:13-6.

68. Callewaert G, Bosteels J, Housmans S, Verguts J, Van Cleynenbreugel B, Van der Aa F, et al. Laparoscopic versus roboticassisted sacrocolpopexy for pelvic organ prolapse: a systematic review. Gynecol Surg 2016;13:115-23.

69. Claerhout F, Verguts J, Werbrouck E, Veldman J, Lewi P, Deprest J. Analysis of the learning process for laparoscopic sacrocolpopexy: identification of challenging steps. Int Urogynecol J 2014;25:1185-91.

70. Mowat A, Maher C, Pelecanos A. Can the learning curve of laparoscopic sacrocolpopexy be reduced by a structured training program? Female Pelvic Med Reconstr Surg 2018;24:272-6.

71. Tan-Kim J, Nager CW, Grimes CL, Luber KM, Lukacz ES, Brown HW, et al. A randomized trial of vaginal mesh attachment techniques for minimally invasive sacrocolpopexy. Int Urogynecol J 2015;26:649-56.

72. Borahay MA, Oge T, Walsh TM, Patel PR, Rodriguez AM, Kilic
GS. Outcomes of robotic sacrocolpopexy using barbed delayed absorbable sutures. J Minim Invasive Gynecol 2014;21:412-6.

73. Kallidonis P, Al-Aown A, Vasilas M, Kyriazis I, Panagopoulos V, Fligou F, et al. Laparoscopic sacrocolpopexy using barbed sutures for mesh fixation and peritoneal closure: a safe option to reduce operational times. Urol Ann 2017;9:159-65.

74. Guan X, Ma Y, Gisseman J, Kleithermes C, Liu J. Robotic single-site sacrocolpopexy using barbed suture anchoring and peritoneal tunneling technique: tips and tricks. J Minim Invasive Gynecol 2017;24:12-3.

75. Liu J, Bardawil E, Zurawin RK, Wu J, Fu H, Orejuela F, et al. Robotic single-site sacrocolpopexy with retroperitoneal tunneling. JSLS 2018;22:e2018.00009.

76. Matanes E, Lauterbach R, Mustafa-Mikhail S, Amit A, Wiener Z, Lowenstein L. Single port robotic assisted sacrocolpopexy: our experience with the first 25 cases. Female Pelvic Med Reconstr Surg 2017;23:e14-8.

77. Lowenstein L, Matanes E, Burke YZ. Surgical technique for robot-assisted sacrocolpopexy performed via a single port. Urology 2017;103:272.

78. Kim S, Pollock GR, Twiss CO, Funk JT. Surgery for posterior compartment vaginal prolapse: graft augmented repair. Urol Clin North Am 2019;46:87-95.

79. Paraiso MF, Barber MD, Muir TW, Walters MD. Rectocele repair: a randomized trial of three surgical techniques including graft augmentation. Am J Obstet Gynecol 2006;195:1762-71.

80. Mowat A, Maher D, Baessler K, Christmann-Schmid C, Haya N, Maher C. Surgery for women with posterior compartment prolapse. Cochrane Database Syst Rev 2018;3:CD012975. 